\title{
Investigating introductory student difficulties reading equipotential diagrams
}

\author{
Rebecca Rosenblatt, Raymond Zich, Amber Sammons, and Jacob Cermak \\ Department of Physics, Illinois State University, Normal, IL 61790
}

\begin{abstract}
This study investigated student difficulties reading equipotential diagrams and the effect a visual change to these diagrams had on students' ability to interpret these diagrams. Equipotential diagrams are often drawn with a uniform line thickness and color. We modified the equipotential diagrams to use color variation and line thickness to indicate the sign and strength of the potential. These changes, which are consistent with theories of visual attention and grounded cognition, exploit students' innate ability to perceive color and line thickness variations to communicate the electric potential. Students compared electric potentials between indicated points on given traditional or modified diagrams. Results show that these students, even after instruction, responded with a distance only electric potential rule. In addition, we found that the modified diagrams did not have the predicted positive effect on students' ability to rank electric potentials; we suspect this is because students' rule for electric potential did not account for charge sign.
\end{abstract}

\section{INTRODUCTION}

This study presents the results of an investigation into students' understanding of the electric potential due to sets of point charges. Students' understanding of the electric potential was assessed by their skill at ranking the electric potential values for selected points in equipotential diagrams. Quantitative and qualitative response data, the effects of two comparative trainings, and eye tracking data of gaze time on certain features of the diagrams are all presented to explain the specific issues that general education students have with interpreting equipotential diagrams for point charges. In addition, the effects of color modifications to traditional equipotential diagrams - created based on theories of visual attention and grounded cognition to support increased student understanding through enhanced visual perception - is investigated.

Previous investigations have found several difficulties students have with electric potential. Students have issues connecting electric potential and current flow, issues with electric potential energy, and issues with rates of change as relating electric potential to electric field [1-3]. Also, many other student difficulties surrounding electric potential have been explored, such as the electric potential for conductors and the choice of the zero point [4]. Separately, there has been extensive research on students' difficulties with common diagrams in physics such as graphs, free-body diagrams, motion maps, etc.. [5]. However, there is surprisingly little research on students' skills working with simple pre-drawn equipotential diagrams, with a 2017 PERC proceedings being one of the few articles the authors found [6].

In addition, there has been some recent work, originating from grounded cognition, on ways to improve physics diagrams so that the visual representations used are perceived in a way that supports the construction of the correct physical model $[7,8]$. On a basic level grounded cognition theory says that when we think about an abstract concept we activate our perceptual system, i.e. sensory motor system, as a way to help us mentally create, or symbolize, the concept. From a diagrammatic perspective, this means that the relevant features of the diagram should be perceivable in a way that supports a correct physical conclusion [5, 7-9]. Here we used equipotential line thickness and red/blue color variations to convey more, or less, positive/negative electric potential.

\section{METHODOLOGY}

All data presented was collected over a two-week period following instruction on electric fields, electric potential, and electric potential energy in an algebra-based physics course at Illinois State University. This class was predominately life science majors in their junior year and was $48 \%$ female. Students participated in an extra credit quiz session that lasted between 30 - 50 minutes in the physics education research laboratory. This method of recruitment had a $75 \%$ participation rate (52 students in total).

Students who attended these sessions were randomly assigned to participate in one of three conditions. Conditions $\mathrm{A}$ and B were two matched sets of computer-based pretest, training, and posttest questions. The third group of students completed questions with an eye tracker recording students' gaze patterns and dwell times when considering a variety of traditional and modified electric potential diagrams. (The analysis that follows assumes randomized groups.)

The task for all three conditions was for students to correctly rank the electric potentials at two points indicated on the diagrams. Equipotential diagrams for a variety of electric charge arrangements were presented to the students. The charge arrangements given to students included single charges, two charges of equal sign and magnitude, two charges of equal sign but different magnitude, and two charges of equal magnitude and opposite sign. These arrangements were all created for both positive and negative charges and the points indicated on each picture varied from points on the same equipotential line (both close to each other and far apart), points adjacent to the same charge but on different lines, and points on different lines and closest to different charges. This yielded 70 different diagrams - 35 traditional black and white diagrams and 35 diagrams modified by color and line thickness. (See Figure 1 for four examples.) 
FIG. 1. 70 matched images were created 35 traditional diagrams and 35 modified color diagrams.

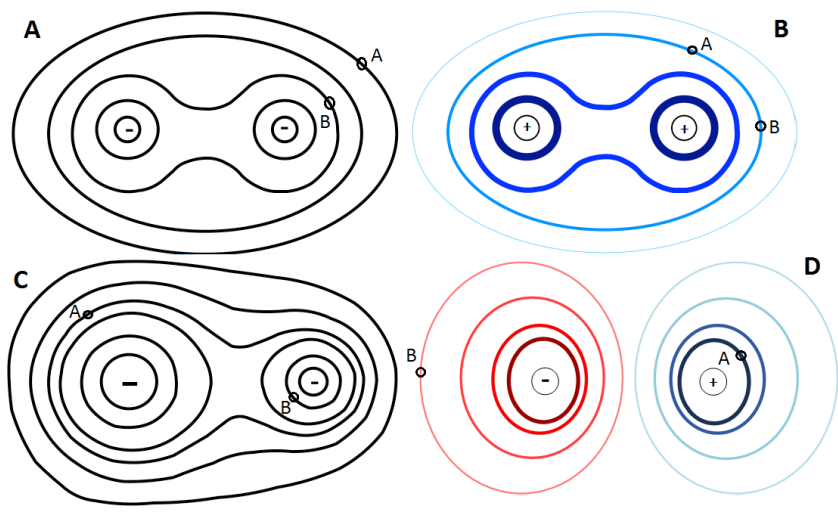

\section{FINDINGS}

\section{A. Pretest Comparisons and Discussion of Item Difficulties}

Condition A gave students 35 questions about traditionally drawn diagrams and then 35 questions with the color modified diagrams. Students then received a short training with traditional diagrams by giving students feedback on the correct answers for 13 items. After this students completed a set of posttest items about the traditionally drawn diagrams.

Condition B gave students 35 questions about color modified diagrams and then gave students 35 questions with the traditionally drawn diagrams. Students then received a short training with color modified diagrams by giving students feedback on the correct answers for 13 items. Students then completed a set of posttest items about the traditionally drawn diagrams which was identical to that of condition A.

The overall scores are listed in Table I by diagram and question set order for conditions $\mathrm{A}$ and B. On average students were $59 \%$ correct on the traditional diagrams and $63 \%$ correct on the color diagrams, and they were at $61 \%$ correct on the first set of 35 questions and $63 \%$ correct on the second set. A two-way anova analysis of these averages shows that there is no main effect due to the type of diagram (traditional vs. modified by color) or the order in which the diagrams were given to the students (first 35 questions or second 35 questions). So, the modification of the diagrams to add color and line thickness did not improve students' correctness, but neither did it make student more likely to be incorrect.

While there is no main effect of diagram or order, there is an interaction between these two $(F=5.149, p=0.028)$. When students were given the traditional diagrams as a first set and then given the new modified diagrams, their answers improved from the first question set by $9 \%$ even though they were not given any feedback on their correctness. However, when students were given the modified (unfamiliar) diagrams first and the traditional diagrams second their correctness decreased by $6 \%$ (see Figure 2).

In addition to considerations of the overall score on the
FIG. 2. Pretest averages show no main effect of diagram type nor question order but do show an interaction.

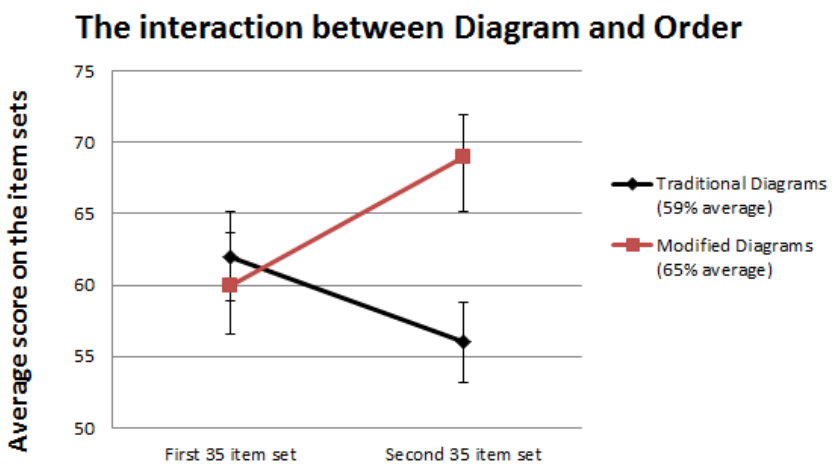

items, an analysis based on the item types shows that students did very well on items that asked about the electric potential when the points were on the same equipotential line and students did well when the charges presented were positive. However, students did very poorly when the charges were negative. An inspection of student responses shows that students were using a response scheme that completely ignored the importance of the sign of the electric charge when reasoning about the electric potential around that charge. If the points selected were on the same equipotential line, students were selecting "equal potential" $82 \%$ of the time, and if one point was on a line closer to a charge, then students were selecting that point as having greater potential $78 \%$ of the time. These two "rules" explain on average $80 \%$ of student pretest responses. Only 4 of the 27 students (15\%) in conditions $\mathrm{A}$ and $\mathrm{B}$ responded with the correct variable rule that electric potential is larger closer to a positive charge and farther from a negative charge. These 4 students used the correct rule $88 \%$ of the time when responding to questions.

Thus, $85 \%$ of these students were using a rule for the electric potential which did not account for the charge. In fact, many students specifically said in comments after completing the assignment that they did not realize at first that the charge mattered. This result explains, in part, why the responses for modified color diagrams were not better than responses for traditional diagrams. The color modified diagrams were designed to help students with the concept that closer points

TABLE I. Pretest Data

\begin{tabular}{llcccc}
\hline \multicolumn{5}{c}{ Condition A } \\
Order & Diagram & Negative & Positive & Same Line & Total \\
First 35 & Traditional & $27 \%$ & $85 \%$ & $90 \%$ & $62 \%$ \\
Second 35 & Modified & $20 \%$ & $98 \%$ & $94 \%$ & $69 \%$ \\
\hline \multicolumn{5}{c}{ Condition B } \\
Order & Diagram & Negative & Positive & Same Line & Total \\
First 35 & Modified & $12 \%$ & $88 \%$ & $84 \%$ & $60 \%$ \\
Second 35 & Traditional & $8 \%$ & $90 \%$ & $85 \%$ & $56 \%$ \\
\hline
\end{tabular}


TABLE II. PostTest Data

\begin{tabular}{lcccc}
\hline \multicolumn{5}{c}{ Condition A: Traditional Diagram Training } \\
Item Description & Negative & Positive & Same Line & Total \\
PreTest Average & $23 \%$ & $92 \%$ & $92 \%$ & $64 \%$ \\
PostTest Average & $83 \%$ & $51 \%$ & $94 \%$ & $75 \%$ \\
\hline \multicolumn{5}{c}{ Condition B: Color Modified Training } \\
Item Description & Negative & Positive & Same Line & Total \\
PreTest Average & $9 \%$ & $89 \%$ & $85 \%$ & $58 \%$ \\
PostTest Average & $79 \%$ & $48 \%$ & $90 \%$ & $71 \%$ \\
\hline
\end{tabular}

were greater magnitude of potential via line thickness and that negative and positive charges had different signs to the potential via changed color. However, students did not have significant pretest issues with distance and did not believe the charge mattered. Thus, the perceived visual stimulus did not work to the students' advantage.

\section{B. The Results of the Short Feedback Based Training}

After students completed the initial pretest question sets, they were given 13 items that displayed the correct answer after they had entered their answer. Condition A received this feedback with traditionally drawn diagrams and group B with the color modified diagrams. All students then did the same set of 35 traditionally drawn posttest items. A paired samples t-test on pre vs. post scores showed a modest overall improvement of $9.75 \%(t=2.834, p=0.009$, effect size 0.5$)$ for both training sets A and B. (Once again, the color modified diagrams did not show either increased nor decreased correctness over the traditional diagrams.)

While the overall pre to post scores showed a modest gain with training, comparing negative charge items and positive charge items showed a more interesting story. Students improved on items which included negative charges by 60 and $70 \%$ respectively for training $\mathrm{A}$ and $\mathrm{B}$, but their scores dropped on items with positive charges by $41 \%$ in both trainings. Thus, it is clear that the training's effect was to get some students to change their response pattern from "the electric potential is greater closer to the charge" to "the electric potential is smaller closer to the charge". In other words, many students still were not recognizing that they needed to have a conditional rule that incorporated the sign of the charge. After training, 7 of the 27 students were using a "the electric potential is smaller closer to the charge" response rule and they used this rule $91 \%$ of the time. After the training, 8 of the 27 students responded with the correct rule $93 \%$ of the time. An additional 7 students used the correct rule most often, but they did so sporadically - $68 \%$ of the time.

\section{Comparing Student Responses to their Rule(s)}

The previous section considered only students' responses to the items and made inferences as to the students mental rule. Here students' posttest scores are compared to students' written responses to three free response questions: 1) Can you give a general rule (or rules) for how you decide if point A has a potential that is greater, less than, or equal to point B? 2) How does your rule (rules) account for the sign of the point charges? 3) Are there any exceptions to your rule (rules)?

Students were coded as having the completely correct rule only if their rule specifically indicated rules for the positive and negative charges. For example: "They were equal when they were on the same electric field line and the same distance away from the + and - poles. The point that was farthest away from the negative charge and closest to the positive had the greater potential." (Note the incorrect term for the equipotential line is used here but this student's response is coded as correct because it accounts for charge and distance.)

Students were coded as having a distance-only negative rule when they gave a response like, "At first, I thought that the closer you are to the charge the greater the potential. I found out later that it was the opposite," or "The electric potential increases as the point gets further from the nucleus."

The distance-only positive rule was coded for responses like, "The further away from the charges the point was the less it was. (The student drew an example with a negative point charge.) If the charges were the same distance then I considered them equal. I treated both charges the same."

For traditional diagrams 10 students wrote a set of rules which accounted for charge sign, distance, and specifically indicated the dichotomy of distance and charge, i.e. the potential was greater farther from a negative charge and greater closer to a positive charge. Of these 10 students, 4 answered questions about negative, positive, and equipotential lines above $90 \%$ correct. 5 of these students answered the negative charge questions and the equipotential questions above $90 \%$ correct but failed to apply the rule they had stated correctly with averages of $(9,18,36,54,63)$ on the positive charge questions. One student answered both the positive and negative questions with medium correctness indicating he/she was sometimes applying the stated correct rule and sometimes applying the incorrect rule. (See Table III for a summary.)

In addition, 3 students stated a distance-only negative rule, i.e. closer is lower potential. These students applied this rule in their answer choices in a remarkably consistent fashion having a correct average of $100 \%$ for negative charge questions and $0 \%$ for positive charge questions. Similarly, 2 students stated a distance-only positive rule, i.e. closer is greater potential with an average of $4 \%$ correct for negative charge questions and $100 \%$ for positive charge questions.

When this same analysis is completed for students in the modified diagram group, results are very similar. A majority of students' ranking responses agree with their stated rules. A main point of interest is that only 1 student (of 5) who gave a completely correct rule failed to use this rule for the positive 
TABLE III. Stated Rule Compared to Response Correctness

\begin{tabular}{lcccc}
\hline \multicolumn{5}{c}{ Condition A: Traditional Diagram Training } \\
Rule & Negative & Positive & Same Line & N \\
Completely Correct & $93 \%$ & $56 \%$ & $95 \%$ & 10 \\
Distance only Negative Rule & $100 \%$ & $0 \%$ & $84 \%$ & 3 \\
Distance only Positive Rule & $4 \%$ & $100 \%$ & $100 \%$ & 2 \\
\hline \multicolumn{5}{c}{ Condition B: Color Modified Training } \\
Rule & Negative & Positive & Same Line & N \\
Completely Correct & $97 \%$ & $71 \%$ & $94 \%$ & 5 \\
Distance only Negative Rule & $85 \%$ & $20 \%$ & $86 \%$ & 4 \\
Distance only Positive Rule & $0 \%$ & $90 \%$ & $72 \%$ & 1 \\
Backwards Rule & $25 \%$ & $36 \%$ & $100 \%$ & 1 \\
\hline
\end{tabular}

charge questions unlike with the traditional diagram training condition where 6 (of 10) students failed to apply the rule consistently with positive charges. It is possible that the colored diagrams helped these students keep track of when the potential was less and when it was more so that these students were able to be more correct on the posttest.

\section{Considering Student Gaze Times and Response Times}

An eye tracker was used to measure gaze times and patterns for both traditional and modified equipotential diagrams. Total gaze time on the image was analyzed based on students' correctness on the ranking task and whether that image was a traditional or modified diagram. In addition, students' gaze time on the small area enclosing the charge was considered to determine whether there was a connection between students' gaze time on the charge and response correctness.

Despite the fact that many students answered pretest questions as if only the distance to the charge mattered and that this response pattern accounted for around $80 \%$ of students' answer choices, the eye tracking data shows that students do fixate on the relatively small area around the provided center charge, but they do not focus on it for a particularly large amount of time. The average time spent fixated on the charges was $376 \mathrm{~ms}$ per item; in comparison students gaze on the entire image was around $3600 \mathrm{~ms}$ and the text associated with the question around $2500 \mathrm{~ms}$. In addition, the average gaze time on the point charges for images with negative charges and indicated points on different equipotential lines was significantly different than their gaze times on the charge for other images (542 ms for images with negative charge vs 334 $\mathrm{ms}$ for other images, $F=7.614, p=0.007$ ). Therefore, students are looking and thus mentally attending to the sign of the charge even if they ultimately do not respond using it.

There were no significant differences in total image gaze times for colored vs. traditional images nor in charge fixation time between correct and incorrect responses. For traditional images the average fixation time when correct was $442 \mathrm{~ms}$ and when incorrect $324 \mathrm{~ms}$. Conversely for the colormodified images (which do not actually require students to fixate on the charge if they are using the color coding) was $335 \mathrm{~ms}$ when correct and $403 \mathrm{~ms}$ when incorrect. This data suggests that visual attention within the traditional diagrams is not a cause for student correctness. This explains why modifying the diagram to direct students' visual attention did not create any differences to students' responses.

\section{CONCLUSION}

This investigation into students' issues interpreting simple equipotential diagrams yielded four main results. First, it demonstrates unambiguously that even after instruction $80 \%$ of these algebra-based physics students do not understand negative electric potential. Second, promoting visual attention to a physically relevant part of the equipotential diagram did not lead to more correct student responses. The eye tracking data and students' pretest responses show that students look at the charges but do not consider them when they rank electric potential because they use a distance only rule. Thus, drawing students' attention to the charge sign with color even though this change is supported by visual attention and grounded cognition theories - was not helpful. This supports other researchers' findings such as Fedlund et. al's paper on the "disciplinary affordances" of diagrams [5]. Third, it illustrates students' issues learning conditional rules. A short training caused about a third of students to shift from incorrectly applying one sometimes-correct rule to everything, to incorrectly applying another sometimes-correct rule to everything. Fourth, because students' response choices and their stated rule(s) are in strong agreement, we recommend quantitative assessments of this topic since this data is easier to collect and score.
[1] A. Leniz, K. Zuza, J. Guisasola, PR-PER, 13, 010128 (2017).

[2] B. A. Lindsey, PRST-PER, 10, 013101 (2014).

[3] R. Allain, Thesis North Carolina State University (2001).

[4] R. L. C. Hazelton, M. R. Stetzer, P. R. L. Heron, P. S. Shaffer, AIP Conference Proceedings 1513, 166 (2013).

[5] T. Fredlund, C. Linder, J. Airey, and A. Linder, PR-PER, 10, 020129 (2014).
[6] E. Gire, A. Wangberg, and R. Wangberg, PERC Proceedings (2017).

[7] Z. Chen, G. Gladding, PRST-PER, 10, 010111 (2014).

[8] L. W. Barsalou, Top. Cog. Sci. 2, 716-724 (2010).

[9] D. Pecher, I. Boot, S. Van Dantzig, In B. Ross (Ed.). The Psychology of Learning and Motivation, 54, 217 (2011).

[10] M. A. Just and P. A. Carpenter, Cognitive Psychology, 8 (4), 441-480 (1976). 